\title{
Study of Thermal Effects Induced by Laser Radiation Incident in an Amplifier: (I) Linear Case
}

\author{
A. F. Hasssan, M. M. El-Nicklawy, S. L. Diab, S. El-Din Salman \\ and A. M Abd-Rabou
}

Helwan University, Faculty of Science, Physics department

\begin{abstract}
The present work aims to study the temperature distribution in a ruby rod pumped in radial direction from a pulsed source located in one focal axis of a cylinder of elliptical cross section while the rod is located in the other one. The study is a function of the focusing parameter of the pump radiation and the half width of a Gaussian distributed intensity incident in axial direction on the amplifying rod. The maxima of the pump radiation and the laser pulse to be amplified are assumed to coincide temporarily. The results show that the temperature in the rod depends on the focusing parameter and the half width of the laser radiation. As the amplification factor was considered to have a small slope around $r=0$, the radial temperature distribution exhibits a dip around $r=0$.
\end{abstract}

\section{Introduction:}

Since the development of lasers several devices were developed allowing its applications in science, engineering and medicine. Due to its high intensity heat will be generated as a result of absorption inside the active medium in the resonator or when it is penetrating an amplifying or absorbing medium. This fact stimulates the studying of the spatial and temporal temperature distribution in order to enhance precession in machining processes or to avoid damage.

Several authors have tried to solve these problems using different methods under different conditions. So for instance have Jabezynski et al. [1] studied thermal length and thermal aberration in diode pumped lasers. [2-4] have studied the fractional heat load in solid induced in diode pumped Nd:YVO4 laser. Analytical method for thermal layout optimization of multilayer structure solid state devices was the subject studied [5]. Heat and temperature distribution in a cladding pumped Er:Yb co-doped phosphate fibre was investigated [6]. The effect of the temperature on the creation of variation of refractive index as well as mechanical deformation and stress was studied [7]. 
As high output power of laser radiation is required, just for instance for laser machining process, and the output power of the laser oscillator is not great enough the radiation will be guided through an amplifier that has to be pumped. The radiation to be amplified and the pump radiation both heat the amplifier and this may lead to phase change, thermal stresses leading to cracks and variation of refractive index and under circumstances to varying the plane form of the input and output surfaces of the amplifier. These effects may lead to damage the amplifier or varying the front surface of the radiation leading to inaccurate laser machining.

To avoid these changes a pre-study of the spatial and temporal temperature distribution has to be carried out. The results of this study will be later applied in equations concerning the thermal stresses, which will be necessary to determine the variation of the refractive index and thus to the deformation of the wave front.

\section{Theory.}

Assuming a homogenous isotropic cylindrical shaped amplifier medium with circular cross section to be air cooled and pumped radialy at its outer surface with a radiation originating from a black body source. The black body and the amplifier medium were located in the two focal axes of a cylinder having elliptical cross section and reflecting outer surface.

The calculated temporal profiles will result from both the pump and the laser radiation to be amplified which are considered to have a Ready temporal distribution [8] with coinciding maxima. Since both pulses are of different pulse durations, the on set of the laser pulse was temporally retarded.

The spatial distribution of the laser radiation which was amplified throughout its propagation along the axis of the amplifier was considered to be a Gaussian shaped radial distribution of different half widths.

To get the temperature distribution within the amplifier medium, the following set of equations is considered.

(1) The heat diffusion equation in cylindrical coordinates assuming rotational symmetry:

(i) Originating from the pump beam:

$$
\frac{\partial^{2} T_{p}(r, t)}{\partial r^{2}}+\frac{1}{r} \frac{\partial T_{p}(r, t)}{\partial r}+\frac{1}{k} g_{p}(r, t)=\frac{1}{\alpha} \frac{\partial T_{p}(r, t)}{\partial t}
$$


(ii) Originating from the laser radiation:

$\frac{\partial^{2} T_{L}(r, z, t)}{\partial r^{2}}+\frac{1}{r} \frac{\partial T_{L}(r, z, t)}{\partial r}+\frac{\partial^{2} T_{L}(r, z, t)}{\partial z^{2}}+\frac{1}{k} g_{L}(r, z, t)=\frac{1}{\alpha} \frac{\partial T_{L}(r, z, t)}{\partial t}$

(2) The boundary condition describing the cooling at the outer radius of the amplifier subjected to the pump process and the radiation to be amplified:

(3) $-\left.k \frac{\partial T_{t}(r, z, t)}{\partial r}\right|_{r=R}=\left.h T_{t}(r, z, t)\right|_{r=R}$

Since the system of the equations governing the temperature distribution is a linear one, therefore the total temperature distribution will be given by:

$$
T_{t}(r, z, t)=T_{L}(r, z, t)+T_{p}(r, t)
$$

where: $T_{p}(r, t)$ Is the temperature distribution generated from the absorbed pump radiation,

$T_{L}(r, z, t)$ Is the temperature distribution generated from the absorbed part of the laser beam to be amplified,

$g_{p}(r, t)$ Is the rate of energy generated from the absorbed part of the pump radiation per unit volume,

$g_{L}(r, z, t)$ Is the rate of energy generated from the absorbed part of the amplified laser radiation per unit volume,

$R$ is the radius of the amplifier,

$\alpha=\frac{k}{\rho C_{p}}$ Is the thermal diffusivity of the amplifier's material,

$\mathrm{h}$ Is the forced convection heat transfer coefficient,

$\mathrm{k}$ Is the thermal conductivity of the amplifier's material,

$\rho$ is the mass density of the amplifier's material and

$C_{p}$ Is the specific heat of the amplifier's material at constant pressure.

The temperature distribution resulting from the pump process was obtained from the Green's function which results from the solution of the homogenous heat diffusion equation subjected to an initial condition. From the obtained Green's function the temperature distribution can be generally calculated from the following equation [9]: 


$$
\begin{aligned}
T(r, t)=\left.\int_{\vec{R}} G\left(\vec{r}, t \mid \vec{r}^{\prime}, \tau\right)\right|_{\tau=0} F\left(\vec{r}^{\prime}\right) d V^{\prime}+\frac{\alpha}{k} \int_{\tau=0} d \tau \int_{\vec{R}} G\left(\vec{r}, t \mid \vec{r}^{\prime}, \tau\right) g\left(\vec{r}^{\prime}, \tau\right) d V^{\prime} \\
+\alpha \int_{\tau=0}^{t} d \tau \sum_{i=1}^{N} \int_{S_{i}} G\left(\vec{r}, t \mid \vec{r}^{\prime}, \tau\right)_{r^{\prime}=r_{i}} \frac{1}{k} f_{i}\left(\vec{r}^{\prime}, \tau\right) d S_{i}^{\prime}
\end{aligned}
$$

where: $\bar{R}$ Is the entire volume of the considered region,

$S_{i}$ Is the boundary surface of region $R, i=1,2,3, \ldots, N$,

$N$ Is the number of continuous surfaces,

$d V^{\prime}$ and $d s_{i}$ are the differential volume and surface element in the $\vec{r}^{\prime}$ variable respectively.

$F\left(\vec{r}^{\prime}\right)$ Is the initial temperature distribution,

$g(\vec{r}, t)$ Represents the contribution of the energy generation leading to heat the amplifier medium,

$G\left(\vec{r}, t \mid \vec{r}^{\prime}, \tau\right)$ Represents the Green's function,

$f_{i}(\vec{r}, \tau)$ Represents the non-homogenous terms of the boundary condition.

Setting $F\left(\vec{r}^{\prime}\right)$ and $f_{i}(\vec{r}, \tau)$ equal to zero, one gets

$$
\left.G_{p}\left(\vec{r}, t \mid \vec{r}^{\prime}, \tau\right)\right|_{\tau=0}=\sum_{m=1}^{\infty} e^{-\alpha \beta_{m}^{2} t} \frac{1}{N\left(\beta_{m}\right)} J_{\circ}\left(\beta_{m} r\right) J_{\circ}\left(\beta_{m} r^{\prime}\right)
$$

Where $J_{\circ}\left(\beta_{m} r\right)$ is the first kind of Bessel function of zero order, $N\left(\beta_{m}\right)$ Is the norm factor and

$\beta_{m}$ Are the eigenvalues of the solution of the heat diffusion equation.

The temperature distribution resulting from the pump beam can thus be given by

$$
T_{p}(r, t)=\frac{\alpha}{k} \int_{\tau=0} d \tau \int_{\bar{R}} G_{p}\left(\vec{r}, t \mid \vec{r}^{\prime}, \tau\right) g_{p}\left(r^{\prime}, \tau\right) d V^{\prime}
$$

With $g_{p}(r, t)=g_{p}(r) G_{p}(t)$,

$$
g_{p}(r)=\frac{R_{\circ}+R}{R_{\circ}+r} e^{\alpha(v)(r-R)}
$$


and

$$
g\left(r^{\prime}, t\right)=g_{p}\left(r^{\prime}, t\right)=X \int \alpha(v) G_{p}(t) I_{o p v}(v) \frac{R_{\circ}+R}{R_{\circ}+r} e^{\alpha(v)(r-R)} d v
$$

Where:

$g_{p}(r)$ Is the radial distribution of the generation function to be determined later on,

$X$ Represents the part of the absorbed radiation which heats the amplifier,

$R_{\text {。 }}$ Is the radius of the focused pump radiation,

$I_{\text {opv }}(v)$ Is the spectral intensity distribution of the pump radiation from the black body source.

The temperature resulting from the absorbed laser radiation is found, after applying the two dimensional Laplace integral transform technique, to be given by:

$T_{L}(r, z, t)=\frac{\alpha}{k} \int_{r=0}^{R} \int_{\tau=0}^{t} r^{\prime} \sum_{m=0}^{\infty} \frac{2 \beta_{m}^{2}}{R^{2}\left(H^{2}+\beta_{m}^{2}\right)} \cdot \frac{1}{J_{\circ}^{2}\left(\beta_{m} R\right)} e^{-\alpha \beta_{m}^{2}(t-\tau)}$

$J_{\circ}\left(\beta_{m} r^{\prime}\right) J_{\circ}\left(\beta_{m} r\right) \int_{0}^{L} G_{L}\left(Z, t \mid z^{\prime}, \tau\right) g_{L}\left(r^{\prime}, z^{\prime}, \tau\right) d r^{\prime} d z^{\prime} d \tau$

with

$$
\begin{aligned}
& \left.G_{L}\left(z, t \mid z^{\prime}, \tau\right)\right|_{\tau=0}=\frac{1}{2 \sqrt{\pi \alpha t}}\left[\exp \left[-\left(\frac{2 L-z-z^{\prime}}{2 \sqrt{\alpha}}\right)^{2} \frac{1}{t}\right]+\exp \left[-\left(\frac{z+z^{\prime}}{2 \sqrt{\alpha}}\right)^{2} \frac{1}{t}\right]+\right. \\
& \quad \exp \left[-\left(\frac{2 L+z-z^{\prime}}{2 \sqrt{\alpha}}\right)^{2} \frac{1}{t}\right]+\exp \left[-\left(\frac{z-z^{\prime}}{2 \sqrt{\alpha}}\right)^{2} \frac{1}{t}\right]+\exp \left[-\left(\frac{2 L+z-z^{\prime}}{2 \sqrt{\alpha}}\right)^{2} \frac{1}{t}\right]+ \\
& \left.\exp \left[-\left(\frac{4 L-z-z^{\prime}}{2 \sqrt{\alpha}}\right)^{2} \frac{1}{t}\right]+\exp \left[-\left(\frac{2 L+z+z^{\prime}}{2 \sqrt{\alpha}}\right)^{2} \frac{1}{t}\right]+\exp \left[-\left(\frac{4 L+z-z^{\prime}}{2 \sqrt{\alpha}}\right)^{2} \frac{1}{t}\right]\right] \\
& g_{L}(r, z, t, v)=\int_{v} I_{o L v}(v, r, 0, t) \alpha_{h} \exp -\left(\alpha_{L}(v, r, t)-\beta_{L}(v, r, t)\right) z d v \\
& I_{o L v}=\tilde{I}_{\circ} g_{L}\left(v, v_{o L}\right) g_{L}(r) G_{L}(t) \text { and }
\end{aligned}
$$


$g_{L}\left(v, v_{o L}\right)=\frac{1}{\Delta v_{o L}} \sqrt{\frac{4 \ln 2}{\pi}} \exp \left(4 \ln 2\left(\frac{v-v_{o L}}{\Delta v_{o L}}\right)\right)$
$\beta_{L}(v, r, t)-\alpha_{L}(v, r, t)=\frac{c^{2} A_{21}}{8 \pi v_{\circ}^{2}} g_{m}\left(v, v_{\circ}\right)\left(N_{2}-\frac{g_{2}}{g_{1}} N_{1}\right)$

Equation (12) will be later developed.

$$
\begin{aligned}
& g_{m}\left(v, v_{\circ}\right)=\frac{1}{\Delta v_{\circ}} \sqrt{\frac{4 \ln 2}{\pi}} \exp \left(-\left(\frac{v-v_{\circ}}{\Delta v_{\circ}}\right)^{2} 4 \ln 2\right) \\
& \left(N_{2}-\frac{g_{2}}{g_{1}} N_{1}\right)=\frac{1}{L}\left(n \ln 10 \sqrt{\frac{\pi}{4 \ln 2}} \frac{8 \pi v_{\circ}^{2} \Delta v_{\circ}}{c^{2} A_{21}}\right)
\end{aligned}
$$

The last equation considers an amplification factor $e^{\left(\beta_{L}(v, r, \tilde{t})-\alpha_{L}(v, r, \tilde{t})\right) L}=10^{n}$ at:

(1) The temporal maximum of the pump radiation $(t=\tilde{t})$,

(2) The end of the amplifier $(\mathrm{z}=L)$

(3) $r=0$ and $R_{\circ}=10^{-3} \mathrm{~m}$.

Where:

$\beta_{L}(v, r, t)$ and $\alpha_{L}(v, r, t)$ are the amplification and attenuation coefficient respectively.

According to this requirement the spectral density of the pump radiation $\rho(v)$, necessary for initiating the required amplification in case of a three level system, is given by

$$
\rho(v)=\frac{A_{21}}{B_{13}} \frac{N_{\circ}+\frac{n \ln 10}{L} \sqrt{\frac{\pi}{4 \ln 2}} \frac{8 \pi v_{\circ}^{2} \Delta v_{\circ}}{c^{2} A_{21}}}{\frac{n \ln 10}{L} \sqrt{\frac{\pi}{4 \ln 2}} \frac{8 \pi v_{\circ}^{2} \Delta v_{\circ}}{c^{2} A_{21}}}
$$

$B_{13}$ Can be obtained from the maxima of the linear absorption coefficient of the two absorbed lines $F_{1}^{4}$ and $F_{2}^{4}$ [10] for the cases of parallel and perpendicular incident radiation. 
Under these conditions the temperature of the pump radiation resulting from the black body source is given by

$$
T=\frac{h v}{k} \frac{1}{\ln \left(\frac{N_{\circ}-\frac{n \ln 10}{L} \sqrt{\frac{\pi}{4 \ln 2} \frac{8 \pi v^{2} \Delta v_{\circ}}{c^{2} A_{21}}}}{N_{\circ}+\frac{n \ln 10}{L} \sqrt{\frac{\pi}{4 \ln 2}} \frac{8 \pi v_{\circ}^{2} \Delta v_{\circ}}{c^{2} A_{21}}} \frac{B_{13}}{A_{21}} \frac{8 \pi h v^{3}}{c^{3}} g_{p}(r=0) G_{p}(\tilde{t})+1\right)}
$$

Thus,

$$
\beta_{L}\left(v_{o L}, r, L, t\right)-\alpha_{L}\left(v_{o L}, r, L, t\right)=\sqrt{\frac{4 \ln 2}{\pi}} \frac{1}{\Delta v_{o L}} \frac{c^{2} A_{21}}{8 \pi v_{\circ}^{2}} N_{\circ} \frac{\frac{B_{13}}{A_{21}} \rho(v) g_{p}(r) G_{p}(t)-1}{\frac{B_{13}}{A_{21}} \rho(v) g_{p}(r) G_{p}(t)+1}
$$

where: $H=\frac{h}{k}$

$N_{1}$ And $N_{2}$ are the densities of the atoms in the lower and upper level respectively,

$g_{1}$ And $g_{2}$ are their statistical weights,

$\Delta v_{\text {。 }}$ Is the full width at half maximum of the spectral gain profile of the laser medium,

$I_{o L v}(v, r, 0, t)$ Is the spectral intensity distribution in interval $v \rightarrow v+d v$ of the laser beam incident in $\mathrm{z}$ direction on the pumped laser rod at $z=0$,

$\alpha_{L}(v, r, t)$ Is the linear attenuation coefficient of the pumped amplifier in the frequency range of the incident laser radiation,

$\beta_{L}(v, r, t)$ Is the linear amplification coefficient of the pumped amplifier medium in the frequency range of the incident laser radiation,

$G_{L}(t)$ Is the time profile of the laser pulse,

$g_{L}(r)$ Is the radial profile of the laser beam,

$g_{m}\left(v, v_{\circ}\right)$ Is the spectral line profile of the medium,

$\alpha_{h}$ Is the part of the linear attenuation coefficient responsible for the heating process and it is equals to $201 / \mathrm{m}$ [10],

$L$ Is the length of the amplified rod and

$g_{L}\left(v, v_{o L}\right)$ Is the normalized spectral profile of the laser radiation,

$\Delta v_{o L} \quad$ Is the full width at half maximum of the laser radiation and

$v_{o L} \quad$ Is the centre frequency of the laser radiation. 


\section{Determination of the heat generation function.}

(a) Resulting from the pump radiation $g_{p}(r, t)$.

Due to the radiation diffraction on the reflecting surface and imperfection of the elliptical cross section, circular spot of radius $R_{\circ}$ with its centre located on the focal axis of the cylinder will be considered. This assumptions leads to the following differential equation for the intensity distribution in redial direction within the amplifier:

$$
\frac{d I_{p}(v, r)}{d r}=-\frac{I_{p}(v, r)}{R_{\circ}+r}+\alpha(v) I_{p}(v, r)
$$

The solution of equation (17), gives with the boundary condition: $I_{p}(v, R)=I_{o p}(v)$

$$
I_{p}(v, r)=I_{o p}(v) \frac{R_{\circ}+R}{R_{\circ}+r} e^{\alpha(v)(r-R)}
$$

Since the pump radiation is considered to be that of a black body source and the absorption spectrum of the amplifier is broad, therefore $I_{p}(v, r)$ and $I_{o p}(v)$ will be replaced by $I_{p v}(v, r) d v$ and $I_{o p v}(v) d v$ respectively.

The generation function responsible for the heating process can be obtained from the first derivative of the exponential factor with respect to $r$ (modified first derivatives), which gives

$$
\int_{v_{1}}^{v_{2}} I_{\text {opv }}(v) d v=g_{p}(r, t)=\int_{v_{1}}^{v_{2}} I_{\text {opv }}(v) \alpha(v) \frac{R_{\circ}+R}{R_{\circ}+r} e^{\alpha(v)(r-R)} d v
$$

where $\alpha(v)$ is the linear attenuation coefficient of the amplifier at the frequency $v$.

(b) Resulting from the laser beam to be amplified through the pumped rod $g_{L}(r, z, t)$.

Assuming the physical parameters of the amplifier to be constant, the differential equation governing the intensity distribution of the laser beam in radial and axial direction might be given by:

$$
\frac{d}{d z} I_{L v}(v, r, z, t)=-I_{L v}(v, r, z, t)\left(\alpha_{L}(v, r, t)-\beta_{L}(v, r, t)\right)
$$


The solution of the last equation gives with the initial condition at $Z=0$ the following solution

$$
I_{L v}(v, r, z, t)=I_{o L v}(v, r, 0, t) \exp \left(-\left(\alpha_{L}(v, r, t)-\beta_{L}(v, r, t)\right) z\right)
$$

\section{Computation.:}

The temperature distribution, resulting from equation (4) after replacing for $T_{p}(r, t)$ and $T_{L}(r, z, t)$ from equation (7) and (9) respectively, was calculated for a Gaussian distributed laser intensity given by

$$
I=I_{\circ} e^{-2 \frac{r^{2}}{w_{0}^{2}}}
$$

Where $w_{\circ}$ is the width of the radiation at which $I_{\circ}$ reduces to $\frac{I_{\circ}}{e^{2}}$.

The time dependence for the pump radiation and the laser beam are given respectively by

$$
\begin{aligned}
& G_{p}(t)=\frac{(n+1)^{n+1}}{n^{n}} \cdot \frac{t}{\Delta t_{p}} \cdot\left(1-\frac{t}{\Delta t_{p}}\right)^{n} \quad n=3 \quad 0 \leq t \leq \Delta t_{p} \\
& G_{L}(t)=\frac{(n+1)^{n+1}}{n^{n}} \cdot \frac{\left(t-\Delta t_{\circ}\right)}{\Delta t_{L}} \cdot\left(1-\frac{\left(t-\Delta t_{\circ}\right)}{\Delta t_{L}}\right)^{n} \quad n=3 \quad 0 \leq t \leq \Delta t_{L}
\end{aligned}
$$

where $\Delta t_{L}$ and $\Delta t_{p}$ are the pulse durations of the laser and pump pulse, respectively, and $\Delta t_{\circ}$ is the time retardation leading to coinciding the two maxima.

\begin{tabular}{|c|c|c|c|c|c|}
\hline Symbol & $\lambda(m)$ & $\rho\left(\mathrm{kg} / \mathrm{m}^{3}\right)$ & $C_{\mathrm{p}}\left(W . S . / \mathrm{kg}^{\circ} \mathrm{K}\right)$ & $N_{\circ}\left(\right.$ ions $\left.m_{3}\right)$ & $v_{01}(\mathrm{~Hz})$ \\
\hline Value & $6943 \times 10^{-10}$ & 4300 & 43 & $1.58 \times 10^{25}$ & $1.5 \times 10^{15}$ \\
\hline Symbol & $v_{21}(\mathrm{~Hz})$ & $v_{22}(\mathrm{~Hz})$ & $v_{31}(\mathrm{~Hz})$ & $v_{32}(\mathrm{~Hz})$ & $F_{\perp}^{21}(1 / m)$ \\
\hline Value & $7.3 \times 10^{14}$ & $7.1 \times 10^{14}$ & $5.4 \times 10^{14}$ & $5.53 \times 10^{14}$ & 320 \\
\hline Symbol & $F_{\perp}^{31}(1 / m)$ & $F_{I I}^{32}(1 / m)$ & $F_{I I}^{01}(1 / m)$ & $F_{I I}^{22}(1 / m)$ & $\Delta v_{\circ}(\mathrm{Hz})$ \\
\hline Value & 155 & 285 & 360 & 280 & $0.329 \times 10^{12}$ \\
\hline Symbol & $k\left(W / m .{ }^{\circ} K\right)$ & $h\left(W / m^{2} .{ }^{\circ} K\right)$ & & & \\
\hline Value & 42 & $1 \times 10^{5}$ & & & \\
\hline
\end{tabular}
The calculation was carried out for $w_{\circ}=10^{-4}, 5 \times 10^{-4} \mathrm{~m}$ and $10^{-3} \mathrm{~m}$ and the following physical and thermal parameters given for ruby in table (1) according to [10].

Table (1): 


\section{Results and Discussion:}

\subsection{Heating resulting from the pump radiation.}

Figure (1) Represents the time dependence of the temperature induced by the pump radiation calculated for $r=0$ and $R$ o as a parameter. From the figure it is evident that the maximum temperature occurs at times greater than $t=\tilde{t}$ which is due to the fact that, up to this time the absorbed radiation overcompensates the heat conduction inside the cooler part of the amplifier. Moreover it is observed that the maximum temperature decreases with increasing $R_{\circ}$ value which matches the fact of the decrease of the intensity at $r=0$ with increasing $R_{\circ}$. The smaller slope of the temperature after switching of the pump radiation with increasing $R_{\circ}$ values is due to the constant temperature in the outer part of the rod (see Fig. (2)).

Figure (2) represents the radial temperature distribution calculated at $t=\tilde{t}$ and $R_{\circ}$ as a parameter. The curves show a relative maximum at $r=0$ this is due to the higher intensity compared with its near surrounding. The decrease of the maximum temperature at $r=0$ and its nearby surrounding with increasing

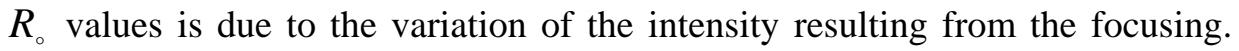
The temperature remains at $r=R$ practically constant. This behaviour is due to the radiation intensity distribution which is in this case equals to $I=I_{o}$.

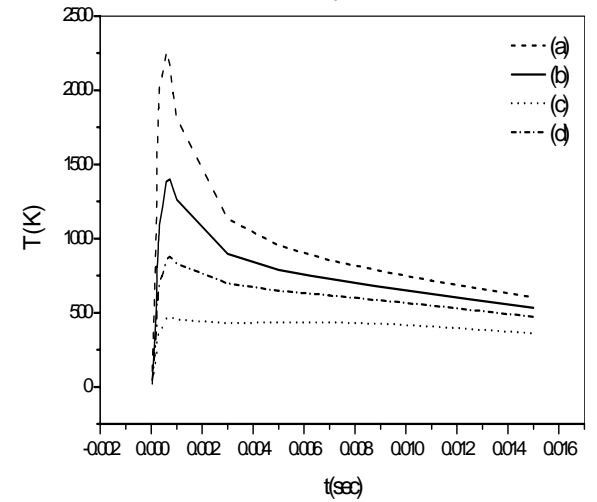

Fig.(1): The temporal temperature distribution induced by the pump radiation calculated for $r=0$ and (a) $R_{\circ}=10^{-4} \mathrm{~m}$ (b) $\mathrm{R}_{\circ}=5 \times 10^{-4} \mathrm{~m}$ (c) $\mathrm{R}_{\circ}=10^{-3} \mathrm{~m}$ (d) $\mathrm{R}_{\circ}=5 \times 10^{-3} \mathrm{~m}$

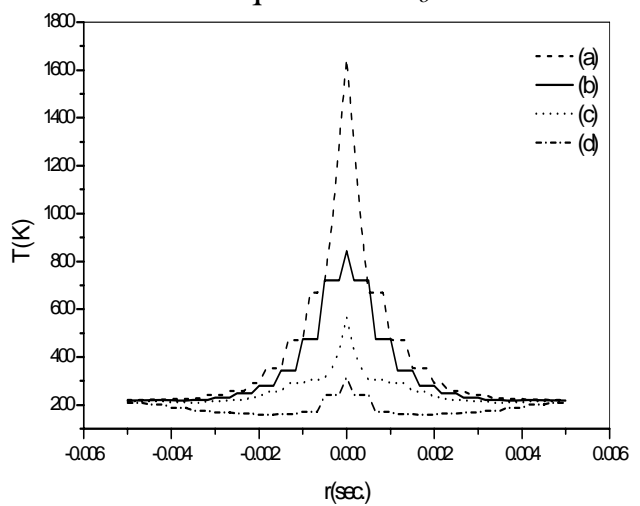

Fig.(2): The radial temperature distribution induced by the pump radiation calculated at $\mathrm{t}=\tilde{\mathrm{t}}$ for (a) $\mathrm{R}_{\mathrm{o}}=10^{-4} \mathrm{~m}$ (b) $\mathrm{R}_{\circ}=5 \times 10^{-4} \mathrm{~m}$ (c) $\mathrm{R}_{\mathrm{o}}=10^{-3} \mathrm{~m}$ (d) $\mathrm{R}_{\mathrm{o}}=$ (d) $5 \times 10^{-3} \mathrm{~m}$

Figure (3) gives the net amplification coefficient, $\left(\beta_{L}-\alpha_{L}\right)$, as a function of the radius of the amplifier calculated at $t=\tilde{t}$. Due to the strong focusing at $R_{\mathrm{o}}=10^{-4} \mathrm{~m}$ the amplification factor at $r=0$ attains its greatest value. 
The negative values of the net amplification coefficient, $\left(\beta_{L}-\alpha_{L}\right)$, appear in a certain range of $r$ in case of $R_{\circ}=5 \times 10^{-3}$ means that absorption takes place.

The appearing of minimum value for the cases $R_{o}=10^{-4} \mathrm{~m}$ and $R_{o}=5^{-4} \mathrm{~m}$ is due to the interaction between the focusing and absorption effects of the pump radiation. The increase of the net amplification after attaining its minimum value for the above cited $R_{o}$ values and the monotone increase for the other $R_{o}$ values is due to the increased intensity of the pump radiation in the outer region of the amplifier.

\section{(II) Heating resulting from the laser radiation.}

Figure (4) represents the temporal temperature distribution calculated for $z=0, r=0$ and all considered $R_{\circ}$ values. The maximum of the temperature occurs at times greater than the maximum of the laser radiation i.e. $t=\tilde{t}$. This behaviour is due to the fact that at that time, the absorbed radiation is equal to the conducted heat. That the temperature distribution is weakly dependent on $R_{\circ}$ is an indicator that the temperature results from the laser radiation absorbed in the vicinity of $z=0$ where practically no amplification takes place and the effect of the greater amplification at greater z-values has not affected the temperature. This behaviour is due to the small time at which the temperature was calculated and the bad heat conductivity of ruby.

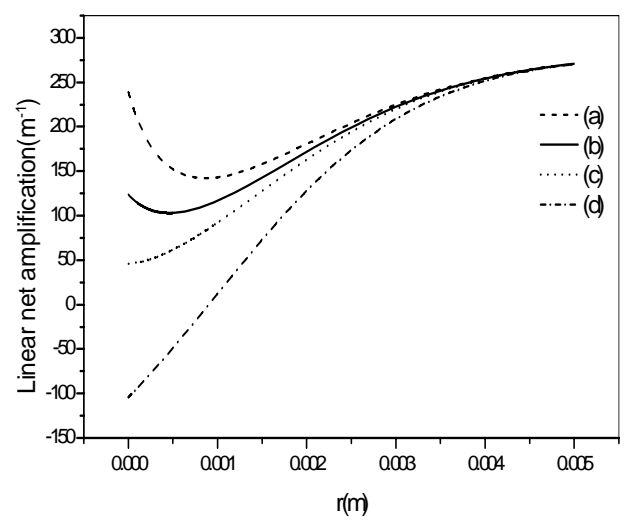

Fig.(3): The radial distribution of the linear net amplification coefficient calculated at $\mathrm{t}=\tilde{\mathrm{t}}$ for (a) $\mathrm{R}_{\mathrm{o}}=10^{-4} \mathrm{~m}$
(b) $\mathrm{R}_{\circ}=5 \times 10^{-4} \mathrm{~m}$
(c) $\mathrm{R}_{\circ}=10^{-3} \mathrm{~m}$
(d) $\mathrm{R}_{\circ}=5 \times 10^{-3} \mathrm{~m}$

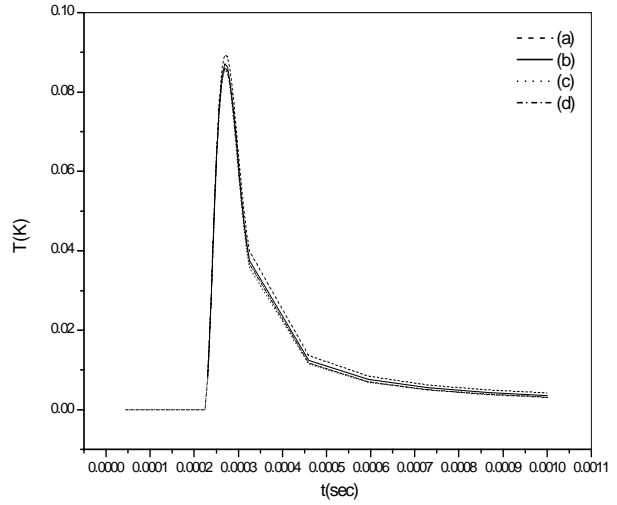

Fig.(4): The temporal temperature distribution induced by a laser beam having a Gaussian intensity distribution of $w_{\circ}=10^{-4} \mathrm{~m}$, and $\Delta t_{L}=10^{-4}$ calculated for $\mathrm{z}=0, \quad \mathrm{r}=0$ and (a) $R_{\circ}=10^{-4} \mathrm{~m}(\mathrm{~b}) \mathrm{R}_{0}=5 \times 10^{-4} \mathrm{~m}$ (c) $R_{\circ}=10^{-3} \mathrm{~m}$ (d) $\mathrm{R}_{\mathrm{o}}=5 \times 10^{-3} \mathrm{~m}$. 
(i) $W_{\circ}=\mathbf{1 0}^{-4} \mathbf{m}$

Figure (5) represents the calculation for the temperature versus time for $z=L, r=0$ and different $R_{\circ}$ values. The figure shows the same temporal behaviour as for $z=0$ except that the temperature values vary drastically with $R_{\circ}$ to reach values between $4 \times 10^{8}$ and $5 \times 10^{-6} \mathrm{~K}^{0}$ for $R_{\mathrm{o}}=10^{-4} \mathrm{~m}$ and $R_{\circ}=5 \times 10^{-3} \mathrm{~m}$ respectively. This behaviour is due to the strong amplification and absorption at the corresponding $R_{\circ}$ values.

Figure (6) represents the temperature distribution along $z$ axis calculated at for $r=0$ and $R_{\circ}$ as a parameter. The curves show an increase of the temperature with increasing $\mathrm{z}$ as there was an amplification (up to $R_{\mathrm{o}}=10^{-3} \mathrm{~m}$ ) and a decrease of the temperature as the net amplification coefficient $\left(\beta_{L}-\alpha_{L}\right)$ turns to be absorptive.

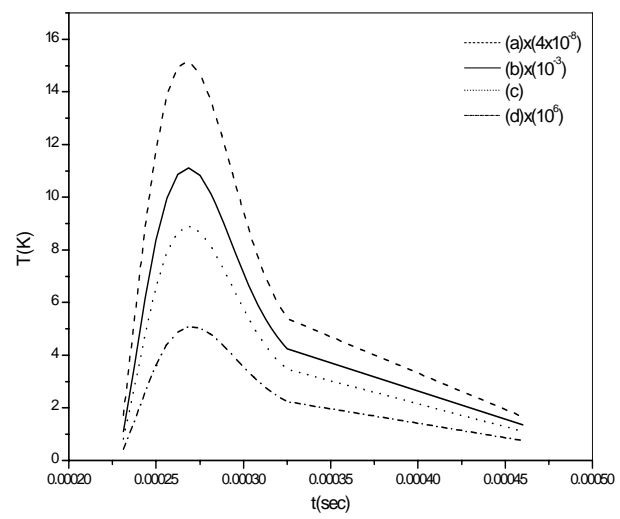

Fig.(5): The temporal temperature distribution induced by a laser beam having a Gaussian intensity distribution of $w_{\circ}=10^{-4} \mathrm{~m}$ and $\Delta t_{L}=10^{-4}$ calculated for $\mathrm{z}=\mathrm{L}, \quad \mathrm{r}=0 \quad$ and $\quad$ (a) $R_{\mathrm{o}}=10^{-4} \mathrm{~m}$ $\begin{array}{lll}\text { (b) } R_{\mathrm{o}}=5 \times 10^{-4} \mathrm{~m} & \text { (c) } R_{\mathrm{o}}=10^{-3} \mathrm{~m} \quad \text { (d) }\end{array}$ $R_{\circ}=5 \times 10^{-3} \mathrm{~m}$

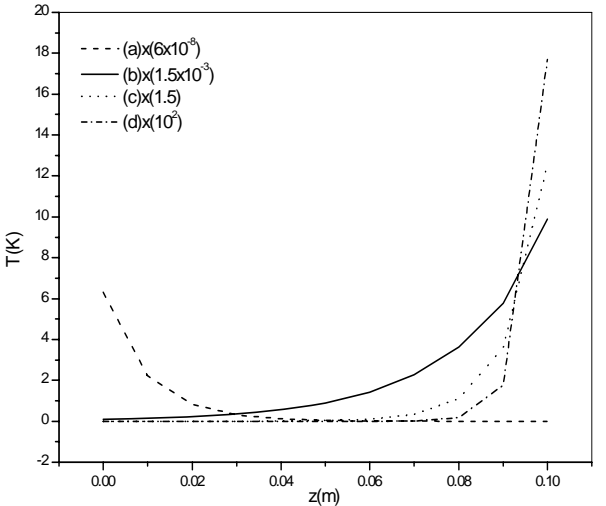

Fig.(6): The axial temperature distribution induced by a laser beam having a Gaussian intensity distribution of $w_{\circ}=10^{-4} \mathrm{~m}$ and $\quad \Delta t_{L}=10^{-4}$ calculated at $t=\tilde{t} \quad$ for $\quad \mathrm{r}=0$ and (a) $R_{\mathrm{o}}=10^{-4} \mathrm{~m}$ (b) $R_{\circ}=5 \times 10^{-4} \mathrm{~m}(\mathrm{c}) R_{\circ}=10^{-3} \mathrm{~m}(\mathrm{~d})$ $R_{\circ}=5 \times 10^{-3} \mathrm{~m}$.

Figure (7) represents the radial distribution of the temperature calculated at $t=\tilde{t}$ for $z=L$ and the considered $R_{\circ}$ values. The figure exhibits a maximum at $r=0$ taking, due to the net amplification coefficient $\left(\beta_{L}-\alpha_{L}\right)$, various values of the temperature ranging between $10^{+8}$ and $10^{-6} \mathrm{~K}$. 
The appearance of a maximum at $\mathrm{r}=0$ for positive $\left(\beta_{L}-\alpha_{L}\right)$ values can be understood from the strong amplification of the laser radiation at $r=0$. But as $\left(\beta_{L}-\alpha_{L}\right)$ becomes negative a maximum at $r=0$ is not logic because of the great absorption at $r=0$ and its neighbourhood. Considering that the value of $\left(\beta_{L}-\alpha_{L}\right)$ does not change drastically with increasing $r$-values compared with the intensity then the appearance of the maximum at $r=0$ can be understood.

(ii) $W_{\circ}=5 \times 10^{-4} \mathrm{~m}$

Fig. (8) represents the temporal temperature distribution calculated for $z=0, r=0$ and all considered $R_{\circ}$ values. Due to the reason given in the discussion of figure (4), the curves are independent of $R_{\circ}$ while the values of the temperature is greater than the corresponding ones in figure (4) due to the greater half width of the laser radiation.

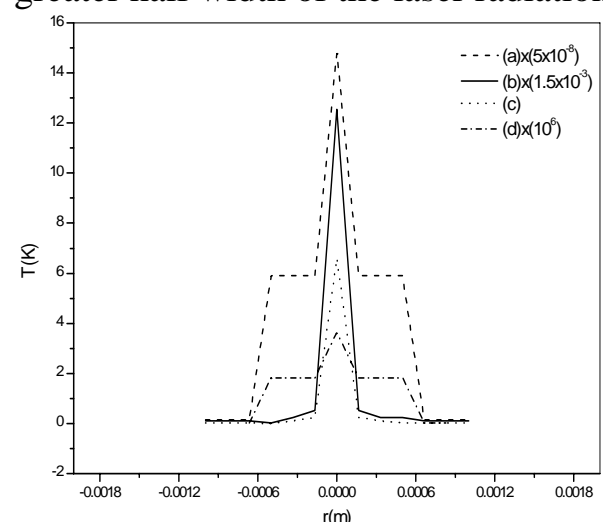

Fig.(7): The radial temperature distribution induced by a laser beam having a Gaussian intensity distribution of $w_{\circ}=10^{-4} \mathrm{~m}$ and $\Delta t_{L}=10^{-4}$ calculated at $t=\tilde{t}$ for $\mathrm{z}=\mathrm{L}$ and (a) $R_{\circ}=10^{-4} \mathrm{~m}$ (b) $R_{\circ}=5 \times 10^{-4} \mathrm{~m}$ (c) $R_{\circ}=10^{-3} \mathrm{~m}$ (d) $R_{\circ}=5 \times 10^{-3} \mathrm{~m}$.

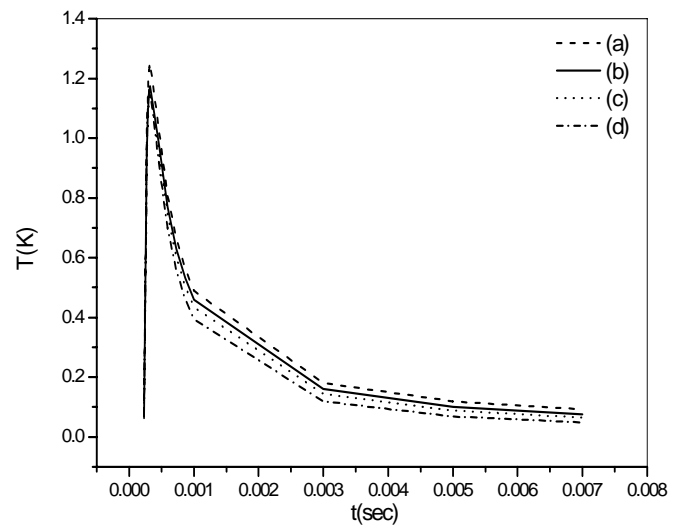

Fig.(8): The temporal temperature distribution induced by a laser beam having a Gaussian intensity distribution of $w_{\circ}=5 \times 10^{-4} \mathrm{~m}$ and $\quad \Delta t_{L}=10^{-4}$ calculated for $\quad \mathrm{z}=0, \quad \mathrm{r}=0 \quad$ and $\quad$ (a) $R_{\mathrm{o}}=10^{-4} \mathrm{~m}$ $\begin{array}{ll}\text { (b) } R_{\circ}=5 \times 10^{-4} \mathrm{~m} & \text { (c) } R_{\circ}=10^{-3} \mathrm{~m}\end{array}$ $R_{\circ}=5 \times 10^{-3} \mathrm{~m}$.

Figure (9) represents the temporal temperature distribution calculated for $z=L, r=0$ and the considered $R_{\circ}$ values. The figure behaves as the corresponding ones in case (i) (Fig. (5)) except that the absolute values of the temperature and the widths are greater. This behaviour is due to the values and distribution of $\left(\beta_{L}-\alpha_{L}\right)$ and the broader half width of the laser spatial distribution. 
Figure (10) Represents the axial temperature distribution calculated at $t=\tilde{t}$ for $r=0$ and the considered $R_{\circ}$ values. The figure behaves as the corresponding curves represented in Fig. (6) except that the temperature, due to the broader half width of the laser profile and the behaviour of $\left(\beta_{L}-\alpha_{L}\right)$, is greater than the corresponding ones represented in Fig. (6).

Figure (11) shows the radial temperature distribution calculated at $t=\tilde{t}$ for $z=L$ and the corresponding $R_{\circ}$ values. The figure shows, due to the broader half width of the laser radiation and as the net amplification coefficient curves becomes monotone increasing; a dip in the radial temperature profile is obtained which is due to the greater amplification or the less absorption in the wings of the radial laser profile than at its central point.

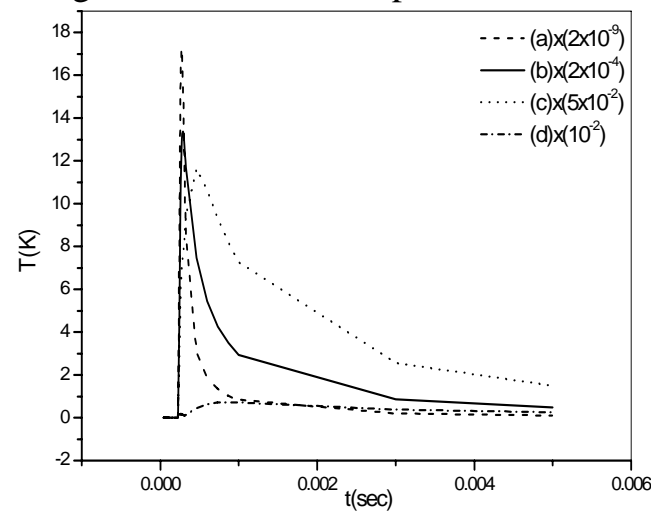

Fig.(9): The temporal temperature distribution induced by a laser beam having a Gaussian intensity distribution of $w_{\circ}=5 \times 10^{-4} \mathrm{~m}$ and

$\Delta t_{L}=10^{-4}$ calculated for $\mathrm{z}=\mathrm{L}, \mathrm{r}=0$ and
(a) $R_{\circ}=10^{-4} \mathrm{~m}$
(b) $R_{\circ}=5 \times 10^{-4} \mathrm{~m}$

(c) $R_{\circ}=10^{-3} \mathrm{~m}$ (d) $R_{\circ}=5 \times 10^{-3} \mathrm{~m}$.

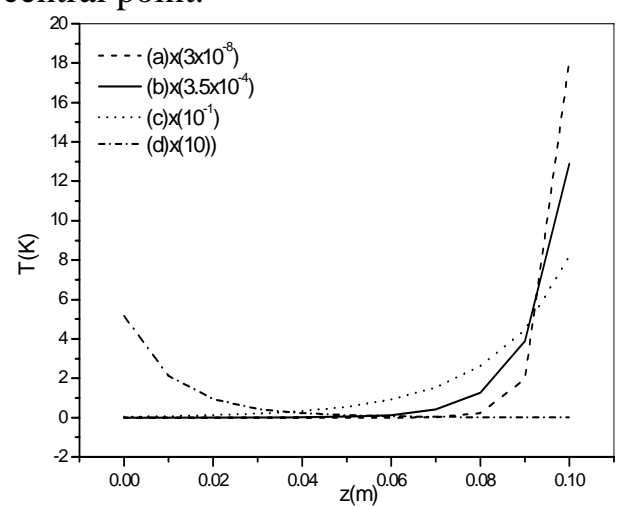

Fig.(10): The axial temperature distribution induced by a laser beam having a Gaussian intensity distribution of $w_{\circ}=5 \times 10^{-4} \mathrm{~m}$ and $\Delta t_{L}=10^{-4}$ calculated at $t=\tilde{t}$ for $\mathrm{r}=0$ (a) $R_{\circ}=10^{-4} \mathrm{~m}$ (b) $R_{\circ}=5 \times 10^{-4} \mathrm{~m}$ (c) $R_{\circ}=10^{-3} \mathrm{~m}$ (d) $R_{\circ}=5 \times 10^{-3} \mathrm{~m}$.

(iii) ${ }^{W_{\circ}}=\mathbf{1 0}^{-3} \mathbf{m}$

Figure (12) represents the temporal temperature distribution calculated for $z=0, r=0$ and all considered $R_{\circ}$ values. The figure shows the same behaviour of figures $(4,8)$ due to the same reasons given in their explanation. Figure (13) Represents the temporal temperature distribution calculated for $z=L$ and $r=0$ with $R_{\circ}$ as a parameter. The figure shows, in accordance with Fig. (5), that the maximum occurs at greater value than $t=\tilde{t}$. Due to the broader half width of the laser radiation, the maximum is greater than the corresponding 
ones of $w_{\circ}=10^{-4} \mathrm{~m}$ and $w_{\circ}=5 \times 10^{-4} \mathrm{~m}$. The difference between these curves and the corresponding ones calculated previously lays in the appearance of the minimum followed by a second maximum. This behaviour can be explained in view of the radial distribution which has higher temperature in the wings. After switch off the laser pulse, the temperature begins to decrease. This decrease will be interpreted by the conduction to the nearby cooler zone. As the time increases an increase of the temperature at $r=0$, resulting from the conducted heat energy from the wings to the central part of the rod, was observed. This behaviour was not observed in the previous case, although the radial distribution has a dip, is due to the very small temperature of the wings which was not able to induce the appearance of the maximum.

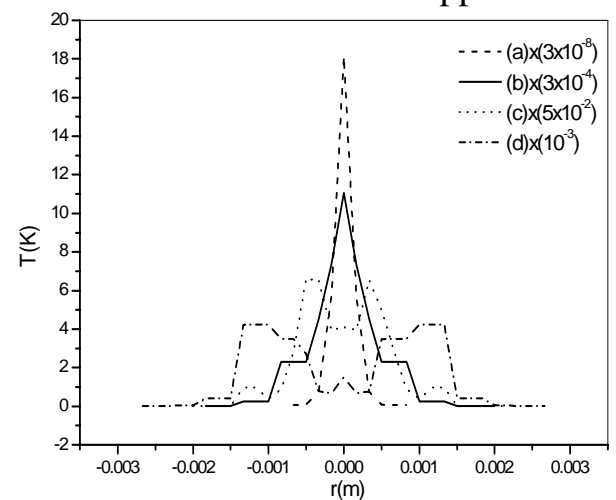

Fig.(11): The radial temperature distribution induced by a laser beam having a Gaussian intensity distribution of $w_{\circ}=5 \times 10^{-4} \mathrm{~m}, \Delta t_{L}=10^{-4}$ calculated at $t=\tilde{t}$ for $\quad \mathrm{z}=\mathrm{L}$ and (a) $R_{\circ}=10^{-4} \mathrm{~m}$ (b) $R_{\circ}=5 \times 10^{-4} \mathrm{~m} \quad$ (c) $R_{\circ}=10^{-3} \mathrm{~m} \quad$ (d) $R_{\circ}=5 \times 10^{-3} \mathrm{~m}$.

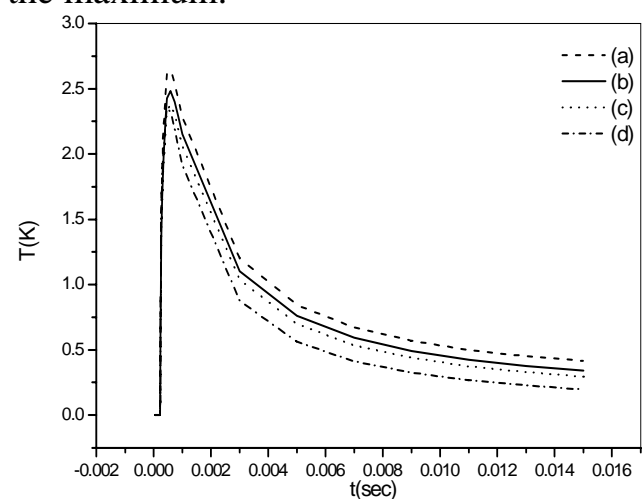

Fig.(12): The temporal temperature distribution induced by a laser beam having a Gaussian intensity distribution of $\mathrm{w}_{\mathrm{o}}=10^{-3} \mathrm{~m}$ and $\Delta t_{L}=10^{-4}$ calculated for $\mathrm{z}=0, \mathrm{r}=0$ and (a) $\mathrm{R}_{\circ}=10^{-4} \mathrm{~m}$ (b) $\mathrm{R}_{\circ}=5 \times 10^{-4} \mathrm{~m}$ (c) $\mathrm{R}_{\mathrm{o}}=10^{-3} \mathrm{~m}$ (d) $\mathrm{R}_{\mathrm{o}}=5 \times 10^{-3} \mathrm{~m}$.

Figure (14) represents the axial temperature distribution calculated at $t=\tilde{t}$ for $r=0$ and the corresponding $R_{\circ}$ values. The increase of the values of the temperature compared with the corresponding curves calculated for $w_{\mathrm{o}}=10^{-4} \mathrm{~m}$ and $w_{\mathrm{o}}=5 \times 10^{-4} \mathrm{~m}$ is the result of the broader spatial half width of the laser radiation. In the contrary to the temperature distribution calculated for $w_{\circ}=10^{-4} \mathrm{~m}$ and $w_{\circ}=5 \times 10^{-4} \mathrm{~m}$ considering $R_{\circ}=5 \times 10^{-3} \mathrm{~m}$ where a monotone decrease of the temperature with increasing $z$ values was observed, the temperature distribution shows in this case a monotone increase with increasing $z$ value. This behaviour can be attributed to the great half width of the laser radiation which will be amplifying to overcompensate the absorption in the vicinity of $r=0$. 
Figure (15) represents the radial distribution calculated at $t=\tilde{t}$ for $z=L$, and $R_{\circ}$ as a parameter. The midpoint of the curves exhibits a maximum which is due to the absorption of the laser radiation that is amplified up to $R_{\circ}=10^{-3} \mathrm{~m}$. As $\left(\beta_{L}-\alpha_{L}\right)$ turns to be negative for $R_{\circ}=5 \times 10^{-3} \mathrm{~m}$ it is due to the value of $\left(\beta_{L}-\alpha_{L}\right)$ which does not change drastically around $r=0$ while the intensity varies exponentially by increasing $r$. For $R_{\circ}=10^{-4} \mathrm{~m}$ no dip was observed this is due to the strong reduction of $\left(\beta_{L}-\alpha_{L}\right)$ as $r$ deviates from zero. For great $R_{\circ}$ values up to $R_{\circ}=10^{-3} \mathrm{~m}$ the positive $\left(\beta_{L}-\alpha_{L}\right)$ and its monotone increase as $r$ increases leading to amplification of the wings of the laser radiation giving a higher temperature for great $r$-values than at $r=0$. For $R_{\circ}=5 \times 10^{-3} \mathrm{~m}$ the negative $\left(\beta_{L}-\alpha_{L}\right)$ values leads to reduction of the temperature at $r=0$, but due to the greater half width of the laser radiation, it attains positive $\left(\beta_{L}-\alpha_{L}\right)$ values great enough to amplify its wings. This behaviour leads to a great temperature at great $\mathrm{r}$ values.

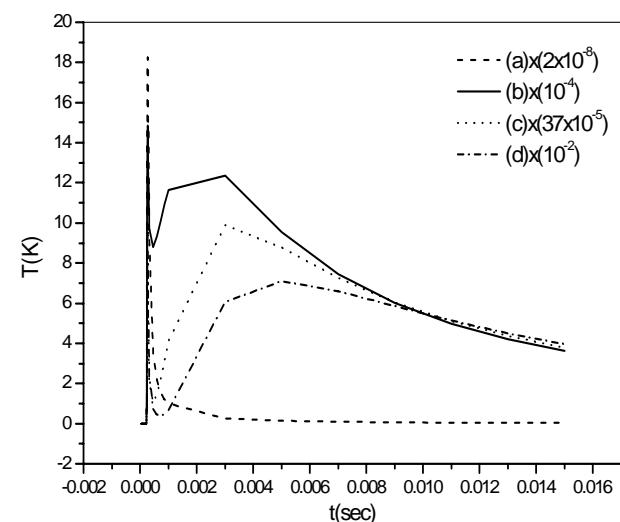

Fig.(13): The temporal temperature distribution induced by a laser beam having Gaussian intensity distribution of $w_{\circ}=10^{-3} \mathrm{~m}$ and $\Delta_{L}=10^{-4}$ calculated for $\mathrm{z}=\mathrm{L} \quad \mathrm{r}=0$ : (a) $\mathrm{R}_{\circ}=10^{-4} \mathrm{~m}$ (b) $\mathrm{R}_{\mathrm{o}}=5 \times 10^{-4} \mathrm{~m}$ (c) $\mathrm{R}_{\mathrm{o}}=10^{-3} \mathrm{~m}$ and (d) $R_{\circ}=5 \times 10^{-3} \mathrm{~m}$.

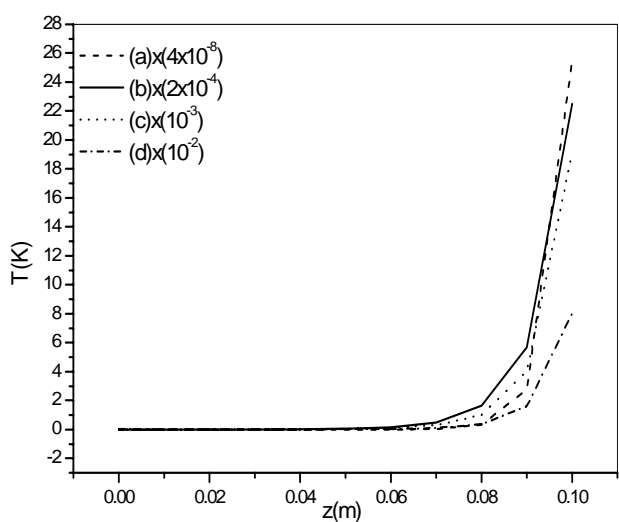

Fig.(14): The axial temperature distribution induced by laser beam having Gaussian intensity distribution of $\mathrm{w}_{\mathrm{o}}=10^{-3} \mathrm{~m}$ and $\Delta t_{L}=10^{-4}$ calculated at $t=\tilde{t}$ for $\mathrm{r}=0$ : (a) $\mathrm{R}_{\mathrm{o}}=10^{-4} \mathrm{~m}$ (b) $\mathrm{R}_{\mathrm{o}}=5 \times 10^{-4} \mathrm{~m}$ and (c) $\mathrm{R}_{\circ}=10^{-3} \mathrm{~m}$ (d) $\mathrm{R}_{\mathrm{\circ}}=5 \times 10^{-3} \mathrm{~m}$. 


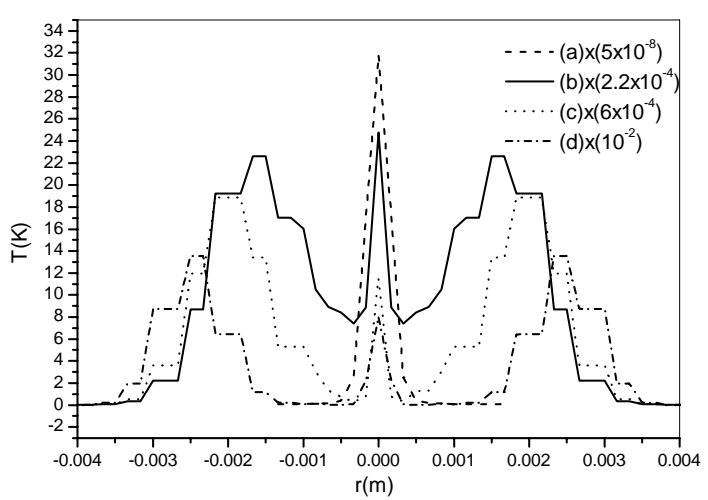

Fig.(15): The radial temperature distribution induced by a laser beam having a Gaussian intensity distribution of $\mathrm{w}_{\mathrm{o}}=10^{-3} \mathrm{~m}$ and $\Delta \mathrm{t}_{\mathrm{L}}=10^{-4}$ calculated at $\mathrm{t}=\tilde{\mathrm{t}}$ for $\mathrm{z}=\mathrm{L}$ and (a) $\mathrm{R}_{\circ}=10^{-4} \mathrm{~m}$ (b) $\mathrm{R}_{\circ}=5 \times 10^{-4} \mathrm{~m}$ (c) $\mathrm{R}_{\circ}=10^{-3} \mathrm{~m}$ (d) $\mathrm{R}_{\circ}=5 \times 10^{-3} \mathrm{~m}$.

\section{References:}

1. JK. Jabczynski, K. Kopcznski and A. Szczesniak, Opt Eng. 35 (12), 3572 (1996).

2. YF. Chen, Kuo H., Opt Lett. 23 (11), 846 (1998).

3. A. Sennanoglu, Appl. Opt. 38 (15), 3253 (1999).

4. K. Pranab, J. Mukhopadhyay, K. George, S. Ranganathan, K. haram and T. Nathan, Optics \& Laser Technology 34, 253 (2002).

5. P. Marcello, G. Agostino and P. Anna Gina, Solid-State Electronics 45, 511 (2001).

6. A. Kosterin, J. Erwin, F. Kevin and M. Mahmoud, Review of Scientific Instruments, 75 (12), 5166 (2004).

7. C. Pfistner, R. Weber, HP. Weber, S. Merrazzi and R. Gruber, IEEE J. Quantum Electron, 30, 1605 (1994).

8. A. F. Hassan , M. M. EL-Nicklawy, M. K. EL-Adawi, E. M. Nasr, A. A. Hemida and O. A. Abed Elgaffar, Optics and Laser technology 28 (5), 337 (1996).

9. M. Necatiozisil "Heat Conduction”, John Wiley \& Sons, INC, New York (1993)

10. W. Koechner "Solid state laser engineering" $4^{\text {th }}$ ed. Berlin Springer, (1992). 